\title{
Comparing Mingoti and Glória's and Niverthi and Dey's multivariate capability indexes
}

\section{Sueli Aparecida Mingoti UFMG}

Fernando Augusto Alves Glória Nestle S/A

\section{ABSTRACT}

In this paper a comparison between Mingoti and Glória's (2003) and Niverthi and Dey's (2000) multivariate capability indexes is presented. Monte Carlo simulation is used for the comparison and some confidence intervals were generated for the true capability index by using bootstrap methodology.

KEY WORDS

Quality control, multivariate capability indexes, Monte Carlo, Bootstrap.

\section{Comparando os coeficientes de capacidade multivariados de Mingoti e Glória e Niverthi e Dey.}

\section{RESUMO}

Neste artigo é apresentada uma comparação entre os índices de capacidade multivariados de Mingoti e Glória (2003) e Niverthi e Dey (2000). O método de simulação de Monte Carlo é utilizado na comparação, e intervalos de confiança para o verdadeiro valor do índice de capacidade do processo são construídos através da metodologia Bootstrap.

PALAVRAS-CHAVE

Controle de qualidade, índices de capacidade multivariados, Monte Carlo, Bootstrap. 


\section{INTRODUCTION}

Process capability indexes (PCI) are used to evaluate the process performance according to the required specifications limits. Some well known indexes for the univariate case are $C_{p}, C_{p k}, C_{p m}$, (MONTGOMERY, 2001; ZHANG, 1998). Very often multiple quality characteristics are used to evaluate the performance of the process and in general they are correlated (MASON; YOUNG, 2002); in these situations, a common procedure is to evaluate the process capability considering each variable separatedly discarding the information of the possible correlation among them. An alternative is to use multivariate capability indexes. The univariate specification interval is then replaced by a specification region and capability indexes are generated according to the joint probability distribution of the variables. In general the multivariate normal distribution is used. Although the multivariate case is very common most of the existing papers in the literature deal with univariate process capability indexes. See Koltz and Johnson (2002) for a good review in the capability subject. Some multivariate PCI's were proposed by Chen (1994), Shahriari et al. (1995) and Taam et al. (1993;1998) using classical statistical estimation procedures. These indexes were compared by Wang et al. (2000) considering some particular examples. Niverthi and Dey (2000) extended the univariate $C_{p}$ and $C_{p k}$ indexes for the case were $p$ quality characteristics are measured in each sample unit; Veever's (1998) introduced a viability index for multiresponse process and Wang (2005) proposed a capability index based upon principal components analysis for short run production. Other interesting references are: Polansky (2001), Foster et al. (2005), Wang (2006), Pearn and Wu (2006) and Pearn et al. (2007). Some PCI's indexes derived under the Bayesian framework are found in Cheng and Spiring (1989), Bernardo and Irony (1996) and Niverthi and Dey (2000) who used Gibbs sampler.

The index proposed by Chen (1994) is quite interesting and it depends on the value of the cumulative distribution function of the maximum coordinate of the random vector $X$ with a $p$-variate normal distribution. However, some analytical or numerical resolution of equations are needed to obtain its value (WANG et al., 2000). By using some ideas suggested in Hayter and Tsui's paper (1994) for correction of control limits in multivariate control charts, Mingoti and Glória (2003) introduced a method which allowed to obtain the numerical value of Chen's capability index using a simulation procedure. This paper presents a comparison of Mingoti and Glória's (2003) with Niverthi and Dey's (2000) indexes by using Monte
Carlo simulation. Some confidence intervals for the true capability indexes were generated by using bootstrap methodology.

\section{UNIVARIATE CAPABILITY INDEXES}

Let $X$ be the quality characteristic of interest with normal distribution with parameters $\mu$ and $\sigma$. Let $L S L$ and USL be the lower and upper specifications limits respectively. The well known capability indexes $C_{p}$ and $C_{p k}$ are defined as

$$
\begin{aligned}
& C_{p}=\frac{U S L-L S L}{6 \sigma} \\
& C_{p k}=\min \left\{\frac{U S L-\mu}{3 \sigma} ; \frac{\mu-L S L}{3 \sigma}\right\}
\end{aligned}
$$

Basically, they represent the relationship between the process and the clients (or project) specification limits. Some references values, such as 1.33 or 2 , are used to classify the process as being capable or not. When $p$ random variables, $p>1$, are monitored at the same time there is a need to build up multivariate indexes. A simple extention of the indexes defined in (1) to the multivariare case is to take the geometric mean of the $C_{p i}$ and $C_{p k i}$ values obtained for each quality characteristic $X_{i}, i=1,2, \ldots, p$. However, this procedure does not take into consideration the relationship that might exist among the variables. Niverthi and Dey (2000) extended the univariate capability indexes, $C_{p}$ and $C_{p k}$, for the multivariate case taking into account the correlation among the variables. Their indexes are basicaly linear combinations of the upper and lower specifications limits of the quality characteristics being the coefficients of the linear combinations related to the covariance matrix of the process. Another alternative was proposed by Chen in 1994 and modified by Mingoti and Glória (2003) who used Hayter and Tsui's (1994) multivariate control limits to build new capability indexes. In the next section Hayter and Tsui's methodology is introduced followed by Chen's, Mingoti and Glória's, Niverthi and Dey's indexes in sections 4,5 and 6 , respectively.

\section{HAYTER AND TSUI CONTROL LIMITS CORRECTION}

Let $X=\left(X_{1} X_{2} \ldots X_{p}\right)$ 'be the random vector with the quality characteristics of interest such that $X$ has a $p$-variate normal distribution with vector mean $\mu^{0}=\left(\mu_{1}^{0} \mu_{2}^{0} \ldots \mu_{p}^{0}\right)$ covariance 
and correlation matrices given by $\Sigma_{p x p}$ and $P_{p x p}$, respectively. According to Hayter and Tsui (1994) for each variable $X_{i}$ the control limits of $(1-\alpha) 100 \%, 0<\alpha<1$, are obtained by choosing a constant $C_{R \alpha}$ which satisfies (2):

$$
\operatorname{Prob}\left\{\left|\frac{X_{i}-\mu_{i}^{0}}{\sigma_{i}}\right| \leq C_{R \alpha}\right\}=1-\alpha, \forall i=1,2, \ldots, p
$$

i.e, the probability that the interval $\left[X_{i} \pm \sigma_{i} C_{R \alpha}\right]$ contains the true value of $\mu_{i}^{0}$ for each $i, i=1,2, \ldots, p$, is equal to $(1-\alpha)$. The choice of the critical value $C_{R \alpha}$ depends upon the correlation matrix $P_{p x p}$ and therefore, the correlation structure of $X$ affects all the intervals simultaneously. The process will be considered out of control if

$$
M=\max \left\{\left|\frac{X_{i}-\mu_{i}^{0}}{\sigma_{i}}\right|, i=1,2, \ldots, p\right\}>C_{R \alpha}
$$

The equation (3) is the maximum of the coordinates of the vector $Z$ which is the vector $X$ standardized. The $C_{R \alpha}$ value is obtained by using a procedure that involves a simulation of samples from a $p$-variate normal distribution with zero mean vector and covariance matrix $P_{p x p}$. In practice the matrix $P_{p x p}$ is estimated by the sample correlation matrix $R_{p x p}$ of $X$ (JOHNSON; WICHERN, 2002). The steps of the simulation algorithm used to obtain the constant $C_{R \alpha}$ is given as below.

Step 1. Generate a large number $N$ of vectors of observations from a $p$-variate normal distribution with mean vector zero and correlation matrix $P_{p x p}$. The generated vectors are denoted by $Z^{1}, Z^{2}, \ldots Z^{N}$.

Step 2. Calculate the statistic $M$ for each one of the generated vectors $Z^{i}=\left(Z_{1}^{i}, Z_{2}^{i}, \ldots, Z_{p}^{i}\right)^{\prime}$ from step 1, i.e, for the every $i=1,2, \ldots, N$, calculate the value of

$$
M^{i}=\max \left\{\left|Z_{j}^{i}\right|, j=1,2, \ldots, p\right\} .
$$

Step 3. Find the value corresponding to the percentil of order $(1-\alpha)$ of the sample $\left(M^{1}, M^{2}, \ldots, M^{N}\right)$ and use the obtained value as the critical value $C_{R \alpha}, 0<\alpha<1$.

This algorithm was also used by Kaldonga and Kulkarni (2004) in control charts for autocorrelated multivariate normal processes. Hayter and Tsui (1994) suggested that a total of $N=100000$ simulations should be performed in order to obtain the value of $C_{R \alpha}$ with high precision. However, Mingoti and Glória (2003) showed that only $N=10000$ is necessary (see also MINGOTI; GLÓRIA,
2005). Hayter and Tsui (1994) also showed that the confidence intervals derived by using the $C_{R \alpha}$ were better than the intervals derived by Bonferroni's method.

\section{CHEN'S MULTIVARIATE CAPABILITY INDEX}

Let $V$ be the specification region of the process defined as

$$
V=\left\{X \in \mathfrak{R}^{p}:\left|X_{i}-\mu_{i}^{0}\right| \leq r_{i}, i=1,2, \ldots, p\right\}
$$

where $\mu_{i}^{0}$ is the specification mean value for the variable $X_{i}$ and $r_{i}$ are the specification constants of the process, $i=1,2, \ldots, p$. Chen's multivariate capability index (1994) is defined as

$$
M C_{p}=\frac{1}{r}
$$

where $r$ is such that

$$
\operatorname{Prob}\left(\max \left\{\left|\frac{X_{i}-\mu_{i}^{0}}{r_{i}}\right|, i=1,2, \ldots, p\right\} \leq r\right)=1-\alpha
$$

The process is considered capable if the value of $M C_{p}$ is larger than 1 and incapable otherwise. Other reference values could be used in place of 1 . The value of $r$ is obtained by using the accumulated distribution function $F_{H}$ of the random variable $H$ which is defined as

$$
H=\max \left(\left|\frac{X_{i}-\mu_{i}^{0}}{r_{i}}\right|, i=1,2, \ldots, p\right)
$$

Therefore, for a certain probability $\alpha, 0<\alpha<1$, the value of $r$ is such that $r=F_{H}^{-1}(1-\alpha)$. Then, if $M C_{p}$ it larger than 1 the process will be considered capable with a certain confidence coefficient $(1-\alpha) 100 \%$. Mingoti and Glória (2003) introduced a modification in Chen's capability index. Instead of using some numerical procedure to find the constant $r$ considering the theoretical distribution of the variable $H$ and equation (5) they proposed to obtain a solution by using the simulation procedure described by the algorithm presented in section 3 . In the next section Mingoti and Glória's modified multivariate $M C_{p}$ index will be presented.

\section{MINGOTI AND GLÓRIA'S MULTIVARIATE CAPABILITY INDICES}

In this section Mingoti and Glória's index will be presented for situations where the process and the nominal mean vectors are equal (section 5.1), the process is not 
centered in the nominal mean vector (section 5.3) and the specification limits are not centered in the nominal mean vector (section 5.2).

\subsection{Processes Centered in the Nominal Vector Mean}

Considering the specification region $V$ defined as in (4) and by using the algorithm described in section 3 , for a fixed value of $\alpha, 0<\alpha<1$, one can find the constant $C_{R \alpha}$ such that

$$
\operatorname{Prob}\left(\max \left\{\left|\frac{X_{i}-\mu_{i}^{0}}{\sigma_{i}}\right|, i=1,2, \ldots, p\right\} \leq C_{R \alpha}\right)=1-\alpha
$$

Therefore the process will be considered capable if for all $i=1,2, \ldots, p$,

$$
\frac{r_{i}}{\sigma_{i} C_{R \alpha}} \geq 1
$$

or equivalently

$$
\frac{\sigma_{i} C_{R \alpha}}{r_{i}} \leq 1
$$

Thus, the multivariate capability index of the process can be defined as

$$
C_{p}^{m}=\max \left\{\frac{\sigma_{i} C_{R \alpha}}{r_{i}}, i=1,2, \ldots, p\right\}
$$

or equivalently,

$$
C_{p}^{m}=\min \left\{\frac{r_{i}}{\sigma_{i} C_{R \alpha}}, i=1,2, \ldots, p\right\}
$$

The process is considered capable if $C_{p}^{m}$ is smaller or equal to 1 , by definition (9), or equivalently if $C_{p}^{m}$ is higher or equal to 1 , by definition (10). The interesting part in this procedure is that there is no need to find the probability distribution of the random variable $Y=\max (Z)$ analytically since the constant $C_{R \alpha}$ is obtained by using a simple simulation routine.

In this paper we will considered the definition (10) for $C_{p}^{m}$.

The procedure described in this section can be implemented in situations where the specification area $V$ is more complex (WANG et al., 2000) and also can be modified for situations where the process does not have the mean vector centered in the nominal value or when the specification limits are not centered in the nominal mean vector as it will be presented in sections 5.3 and 5.2, respectively.

\subsection{More general case: specifications limits not centered in the nominal mean}

Let $L S L_{i}$ and $U S L_{i}$ be the lower and upper specification limits for the quality characteristic $X_{i}, i=1,2, \ldots, p$. The multivariate capability index $C_{p}^{m}$ is then defined as

$$
C_{p}^{m}=\min \left\{C_{p i}^{m}, i=1,2, \ldots, p\right\}
$$

where

$$
C_{p i}^{m}=\left[\frac{U S L_{i}-L S L_{i}}{2 \sigma_{i} C_{R \alpha}}\right], \quad i=1,2, \ldots, p
$$

and $\sigma_{i}$ is the standard deviation of $X_{i}$. The process is considered capable for $C_{p}^{m}$ higher or equal to 1 . If for each variable $X_{i}, i=1,2, \ldots, p$, the specification limits are centered in the nominal mean value then the equation (11) and (10) are equal since $\left(U S L_{i}-L S L_{i}\right)=2 r_{i}$.

\subsection{Processes not centered in the nominal mean}

In many situations the process is in statistical control but is not centered in the specification mean vector. The $C_{p}^{m}$ defined in sections 5.1 and 5.2 are not sensible to changes in the process vector mean and need to be modified. A similar approach as in the derivation of the $C_{p k}$ index in the univariate case can be adopted to define a multivariate coefficient $C_{p k}^{m}$.

Let $L S L_{i}$ and $U S L_{i}$ be defined as in section 5.2 and let $\mu_{i}^{0}$ and $\sigma_{i}$ be the process mean and standard deviation of the variable $X_{i}$. Then the multivariate coefficient $C_{p k}^{m}$ is defined as

$$
\begin{aligned}
C_{p k}^{m} & =\min \left\{\min \left(\frac{\mu_{i}^{0}-L S L_{i}}{C_{R \alpha} \sigma_{i}} ; \frac{U S L_{i}-\mu_{i}^{0}}{C_{R \alpha} \sigma_{i}}\right), i=1,2, \ldots, p\right\}= \\
& =\min \left\{\left(\frac{\mu_{i}^{0}-L S L_{i}}{C_{R \alpha} \sigma_{i}} ; \frac{U S L_{i}-\mu_{i}^{0}}{C_{R \alpha} \sigma_{i}}\right), i=1,2, \ldots, p\right\}
\end{aligned}
$$

Considering that $L S L_{i}=\mu_{i}^{S}-r_{i}^{l}$ and $U S L_{i}=\mu_{i}^{S}+r_{i}^{2}$ where $\mu_{i}^{s}$ is the specification mean of $X_{i}$, and $r_{i}^{1}$ and $r_{i}{ }^{2}$ are constants, the equation (12) reduces to

$C_{p k}^{m}=\min \left\{\frac{\left.\mu_{i}^{0}-\mu_{i}^{s}+r_{i}^{l}\right)}{C_{R \alpha} \sigma_{i}} ; \frac{\left(\mu_{i}^{s}-\mu_{i}^{0}+r_{i}^{2}\right)}{C_{R \alpha} \sigma_{i}}, i=1,2, \ldots, p\right\}$

and therefore it takes into account possible deviations from the process means to the nominal means values. When for each variable $X_{i}=1,2, \ldots, p$, the process is centered in the specification mean value, the $C_{p k}^{m}$ is equal to the value obtained by equation (10) if the specification limits are centered in the nominal means or it is equal to (11) if they are not.

It is important to point out that the indexes $C_{p}^{m}$ and $C_{p k}^{m}$ are the minimum (or maximum) of a vector that has $p$ coordinates each one representing the capability index 
related to the quality characteristic $X_{i}=1,2, \ldots, p$. Therefore, Mingoti and Glória's (2003) indexes quantify the global capability as well as the capability of the process for each quality characteristic individually. If the researcher wants to know which variables are responsible for the global noncapability of the process it will be enough to observe the individual indexes looking for those that are smaller than 1 if $C_{p}^{m}$ and $C_{p k}^{m}$ are defined as a minimum or higher than 1 , if $C_{p}^{m}$ and $C_{p k}^{m}$ are defined as a maximum.

\section{NIVERTHI AND DEY'S MULTIVARIATE PROCESS CAPABILITY INDEXES}

Niverthi and Dey (2000) proposed an extension of the univariate $C_{p}, C_{p k}$, for the multivariate case as follows. Let $X=\left(X_{1} X_{2} \ldots X_{p}\right)$ ' be the vector containing the quality characteristics with a $p$-variate normal distribution with parameters $\mu^{0}=\left(\mu_{1}^{0} \mu_{2}^{0}{ }_{2} \ldots \mu_{p}^{0}\right)^{\prime}$ and $\Sigma_{p \times p}$. Let $U S L=\left(U S L_{1}\right.$ $\left.U S L_{2} \ldots U S L_{p}\right)^{\prime}$ and $L S L=\left(L S L_{1} L S L_{2} \ldots L S L_{p}\right)^{\prime}$ 'be the upper and lower specification vectors, $L S L_{i}$ and $U S L_{i}$ as defined in section $5.2, i=1,2, \ldots, p$. The Niverthi and Dey's multivariate versions of univariate $C_{p}$ and $C_{p k}$ are linear combinations of the upper and lower specifications limits of the $p$ variables and are defined as

$$
\begin{aligned}
& C_{p}^{N D}=\Sigma_{p x p}^{-1 / 2}\left(\frac{U S L-L S L}{2 k}\right) \\
& C_{p k}^{N D}=\Sigma_{p x p}^{-1 / 2}\left(\min \left\{\frac{U S L-\mu^{0}}{k} ; \frac{\mu^{0}-L S L}{k}\right\}\right)
\end{aligned}
$$

In this case a capability value is generated for each quality characteristic, since $C_{p}^{N D}$ and $C_{p k}^{N D}$ are ( $\left.p \times 1\right)$ dimensional vectors. The value of the constant $k$ is based on the univariate standard normal distribution. Niverthi and Dey (2000) used $\mathrm{k}=3$ in (13) which corresponds to an area of $99.73 \%$ or a significance level of $\alpha=0.0027$.

\section{EXAMPLE}

For this example we will use $p=4$ variables of the aircraft data set presented in Niverthi and Dey's paper (2000) which originally has $n=50$ observations related to measurement (in centimeters) on 10 different aircraft features from a component hub which is part of the engine. The production of these parts is made with high degree of precision. The 4 variables presented in this example, according to the original notation of Niverthi and Dey's paper are: MQI128, MQI444, MQI519 and MQI514. The vectors with the specification limits given by Niverthi and Dey (2000) are

$$
\begin{aligned}
L S L & =\left(\begin{array}{llll}
6.393 & 0.594 & 1.852 & 23.677
\end{array}\right)^{\prime} \\
\mu^{S} & =\left(\begin{array}{llll}
6.395 & 0.597 & 1.854 & 23.679
\end{array}\right)^{\prime} \\
U S L & =\left(\begin{array}{llll}
6.397 & 0.600 & 1.856 & 23.681
\end{array}\right)^{\prime}
\end{aligned}
$$

The sample covariance and correlation matrices are respectively given by

$$
\begin{gathered}
S_{4 \times 4}=\left[\begin{array}{cccc}
7.773 e^{-8} & -6.931 e^{-8} & 3.102 e^{-8} & -2.995 e^{-8} \\
-6.931 e^{-8} & 1.326 e^{-6} & -1.102 e^{-7} & 3.392 e^{-8} \\
3.102 e^{-8} & -1.102 e^{-7} & 1.176 e^{-7} & -3.959 e^{-8} \\
-2.995 e^{-8} & 3.392 e^{-8} & -3.959 e^{-8} & 1.420 e^{-7}
\end{array}\right] \\
R_{4 \times 4}=\left[\begin{array}{cccc}
1 & -0.216 & 0.324 & -0.285 \\
-0.216 & 1 & -0.279 & 0.078 \\
0.324 & -0.279 & 1 & -0.306 \\
-0.285 & 0.078 & -0.306 & 1
\end{array}\right]
\end{gathered}
$$

The Niverthi and Dey's (2000) estimated multivariate $C_{p}^{N D}$ for $\alpha=0.0027$ and $\alpha=0.05$ are given respectively by

$\hat{C}_{p}^{N D}=\left[\begin{array}{llll}2.677 & 1.085 & 2.305 & 2.373\end{array}\right]^{\prime}$ (for $\left.\alpha=0.0027\right)(17)$

$\hat{C}_{p}^{N D}=\left[\begin{array}{llll}4.015 & 1.6273 .4573 .559\end{array}\right]^{\prime} \quad($ for $\alpha=0.05)$

Due to the fact that the process is centered in the nominal mean (see Table 1) for each $\alpha$ the estimated value of $\widehat{C}_{p k}^{N D}$ is equal to $\widehat{C}_{p}^{N D}$. There is a difficulty to use $\widehat{C}_{p}^{N D}$ to decide if the process is capable or not because there is no reference values to which the vector $\widehat{C}_{p}^{N D}$ could be compare to. One possibility is to use the usual univariate $C_{p}$ reference value for each variable separatedly. Another one is to define the global capability process estimate as the minimum value of the vector $\widehat{C}_{p}^{N D}$ which for this example is 1.085 (for $\alpha=$ 0.0027 ) and 1.627 (for $\alpha=0.05$ ). It is important to clarify that the $\widehat{C}_{p}^{N D}$ values showed in Niverthi and Dey's paper (p.677) are different than the values in (17) since in their example they used the complete random vector with $p=10$ variables to generate the capability estimation and therefore their covariance and correlation matrices are different than (15) and (16), respectively.

To obtain the value of the Mingoti and Glória's multivariate PCI proposed in section $5.1\left(C_{p}^{m}\right)$ it is necessary to calculate the constant $C_{R \alpha}$. Table 2 presents the values of $C_{R \alpha}$, for 
$\alpha=0.05$, obtained by applying the simulation algorithm described in section 3, for $N=1000,10000$ and 100000 , considering a standard multivariate normal distribution with correlation matrix equals to (16). The corresponding values of $C_{p}^{m}$ (according to equation (10)) are also presented. As one can see the values of $C_{R \alpha}$ for $N=10000$ and $N=100000$ are very similar indicating that in fact, there is no need to perform 100000 simulations as suggested by Hayter and Tsui (1994). For this example the value of $C_{R \alpha}$ was considered as 3.327 for $\alpha=0.0027$ and 2.487 for $\alpha=0.05$. The multivariate process capability $C_{p}^{m}$ is estimated as

$\hat{C}_{p}^{m}=\min \{2.155 ; 0.783 ; 1.757 ; 1.595\}=0.783($ for $\alpha=0.0027)$

$\hat{C}_{p}^{m}=\min \{2.883 ; 1.048 ; 2.347 ; 2.051\}=1.048($ for $\alpha=0.05)$

indicating that the process is incapable at $99.73 \%$ and capable at $95 \%$. However, for this confidence level the estimated $C_{p}^{m}$ is very close to 1 which gives a warning signal.

Table 1: Descriptive statistics - the airplane example.

\begin{tabular}{ccccc}
\hline Variable & Mean & $\begin{array}{c}\text { Standard } \\
\text { Dev. }\end{array}$ & Minimum & Maximum \\
\hline MQI128 & 6.395 & 0.000279 & 6.3944 & 6.3958 \\
MQI444 & 0.597 & 0.001150 & 0.5946 & 0.5994 \\
MQI519 & 1.854 & 0.000343 & 1.8540 & 1.8550 \\
MQI514 & 23.679 & 0.000377 & 23.6780 & 23.6810 \\
\hline
\end{tabular}

Table 2: $\boldsymbol{C}_{\boldsymbol{R} \alpha}$ and $\boldsymbol{C}_{p}^{m}$ values $-\alpha=0.05$.

\begin{tabular}{rrrr}
\hline $\mathrm{N}$ & 1000 & 10000 & 100000 \\
\hline$C_{R \alpha}$ & 2.51392 & 2.48758 & 2.48030 \\
$C_{p}^{m}$ & 1.03628 & 1.04796 & 1.05032 \\
\hline
\end{tabular}

\section{A SIMULATION STUDY}

In this section we present the results of a simulation study. Two processes were considered with parameters and respective specifications limits as given in sections 8.1 and 8.2.

\subsection{Process 1 - centered in the specification vector mean}

Let the mean vector, the specification limits, the covariance and correlation matrices be given as mean vector: $\mu^{0}=[4080]^{\prime}=\mu^{s}$, covariance matrix:
$\Sigma_{0}=\left[\begin{array}{cc}16 & 14.4 \\ 14.4 & 16\end{array}\right]$ correlation matrix: $\rho^{0}=\left[\begin{array}{cc}1 & 0.9 \\ 0.9 & 1\end{array}\right] ; U S L=\left[\begin{array}{ll}60 & 98\end{array}\right] ; \quad L S L=$
$[2062]^{\prime}$.

It represents a situation where there is a high correlation between the two quality characteristics. For $\alpha=0.0027$ the true univariate capability indexes are: $C_{p 1}=1.66$ and $C_{p 2}=1.5$ and the geometric mean is equal to 1.578 . The multivariate Niverthi and Dey's is given by:

$C_{p}^{N D}=\frac{1}{6}\left[\begin{array}{cc}16 & 14.4 \\ 14.4 & 16\end{array}\right]^{\frac{-1}{2}}\left[\begin{array}{c}60-20 \\ 98-62\end{array}\right]=\left[\begin{array}{cc}0.48 & -0.3046 \\ -0.3046 & 0.48\end{array}\right]$

$$
\left[\begin{array}{l}
40 \\
36
\end{array}\right]=\left[\begin{array}{l}
1.412 \\
0.885
\end{array}\right]
$$

with minimum equals to 0.885 . For $\alpha=0.0027$ the constant $C_{R \alpha}$ is 3.149 and Mingoti and Glória's index is given by

$C_{p}^{m}=\min \left\{\frac{20}{(3.149) 4} ; \frac{18}{(3.149) 4}\right\}=\min \{1.588 ; 1.429\}=1.429$

For $\alpha=0.05$ the values of the univariate capability indexes are: $C_{p 1}=2.5, C_{p 2}=2.25$ and the geometric mean is equal to 2.37. The multivariate Niverthi and Dey's is given by

$$
C_{p}^{N D}=\frac{1}{4}\left[\begin{array}{cc}
0.48 & -0.3046 \\
-0.3046 & 0.48
\end{array}\right]\left[\begin{array}{l}
40 \\
36
\end{array}\right]=\left[\begin{array}{l}
2.118 \\
1.328
\end{array}\right]
$$

with minimum equals to 1.328 . The constant $C_{R \alpha}$ for $\alpha=0.05$ is 2.092 and Mingoti and Glória's is given by

$C_{p}^{m}=\min \left\{\frac{20}{(2.092) 4} ; \frac{18}{(2.092) 4}\right\}=\min \{2.390 ; 2.151\}=2.151$

Figure 1 shows the specification and confidence regions for 99.73 and $95 \%$ considering a bivariate normal distribution of process 1 . It is very clear that this process is capable. However, for $99.73 \%$ Niverthi and Dey's suggests that the process is not capable in the second variable. The geometric means indicate that the process is capable for both $\alpha$ values. Comparing to Niverthi and Dey's in this example, Mingoti and Glória's index represented better the global capability of the process.

\subsection{Process 2 - not centered in the specification mean vector}

Let the mean vector, the specification limits, the covariance and correlation matrices be given as 
mean vector: $\mu^{0}=\left[\begin{array}{ll}50 & 85\end{array}\right]$; covariance matrix: $\Sigma^{0}=\left[\begin{array}{ll}4 & 2 \\ 2 & 4\end{array}\right]$;
$\mu^{s}=\left[\begin{array}{ll}40 & 80\end{array}\right] ;$ correlation matrix: $\rho^{0}=\left[\begin{array}{cc}1 & 0.5 \\ 0.5 & 1\end{array}\right] ; U S L=\left[\begin{array}{ll}60 & 90\end{array}\right] ; L S L=$
$[20$ 70]'.

It represents a situation where there is a moderate correlation between the two quality characteristics. For $\alpha=0.0027$ the true univariate capability indexes are: $C_{p 1}=1.66, C_{p 2}=0.833$ and the geometric mean is equal to 1.176 . The multivariate Niverthi and Dey's is given by the vector

$$
\begin{aligned}
C_{p k}^{N D}= & {\left[\begin{array}{ll}
4 & 2 \\
2 & 4
\end{array}\right]^{\frac{-1}{2}}\left\{\left[\begin{array}{l}
\min \left(\frac{10}{3} ; \frac{30}{3}\right) \\
\min \left(\frac{5}{3} ; \frac{15}{3}\right)
\end{array}\right]\right\}=\left[\begin{array}{cc}
0.5577 & -0.1495 \\
-0.1495 & 0.5577
\end{array}\right] } \\
& {\left[\begin{array}{c}
\frac{10}{3} \\
\frac{5}{3}
\end{array}\right]=\left[\begin{array}{l}
1.609 \\
0.431
\end{array}\right] }
\end{aligned}
$$

with minimum equals to 0.431 . For $\alpha=0.0027$ the constant $C_{R \alpha}$ is 3.195 and Mingoti and Glória’s capability index is given by

$$
\begin{aligned}
C_{p k}^{m} & =\min \left\{\min \left(\frac{30}{(3.195) 2} ; \frac{10}{(3.195) 2}\right) ; \min \left(\frac{15}{(3.195) 2} ; \frac{5}{(3.195) 2}\right)\right\} \\
& =\min \{1.565 ; 0.783\}=0.783
\end{aligned}
$$

For $\alpha=0.05$ the values of the univariate capability indexes are: $C_{p 1}=2.49, C_{p 2}=1.249$ and the geometric mean is equal to 1.764 . The multivariate Niverthi and Dey's is given by the vector

$$
\begin{aligned}
C_{p k}^{N D}= & {\left[\begin{array}{ll}
4 & 2 \\
2 & 4
\end{array}\right]^{\frac{-1}{2}}\left\{\left[\begin{array}{l}
\min \left(\frac{10}{2} ; \frac{30}{2}\right) \\
\min \left(\frac{5}{2} ; \frac{15}{2}\right)
\end{array}\right]\right\}=\left[\begin{array}{cc}
0.5577 & -0.1495 \\
-0.1495 & 0.5577
\end{array}\right] } \\
& {\left[\begin{array}{c}
\frac{10}{2} \\
\frac{5}{2}
\end{array}\right]=\left[\begin{array}{l}
2.415 \\
0.647
\end{array}\right] }
\end{aligned}
$$

with minimum equals to 0.647 . For $\alpha=0.05$ the constant $C_{R \alpha}$ is 2.198 and Mingoti and Glória's capability is given by

$$
\begin{aligned}
C_{p k}^{m} & =\min \left\{\min \left(\frac{30}{(2.198) 2} ; \frac{10}{(2.198) 2}\right) ; \min \left(\frac{15}{(2.198) 2} ; \frac{5}{(2.198) 2}\right)\right\} \\
& =\min \{2.275 ; 1.137\}=1.137
\end{aligned}
$$

Figure 2 shows the specification and confidence regions for 99.73 and $95 \%$ considering the bivariate normal distribution of process 2. It is clear that this process is not capable for $99.73 \%$ as both multivariate capability indexes indicated. For 95\% Niverthi and Dey's suggests that the process is not capable in the second variable but the confidence region is still inside the specification region although very close to the upper specification limit for the second variable. Mingoti and Glória’s index indicated a warning signal since it resulted in a value very close to 1 . By the geometric means the process is considered capable for both confidence levels although for $99.73 \%$ the estimated value is very close to 1 .

It is important to point out that in both situations, process 1 and 2, the geometric mean resulted in higher values than both multivariate indexes $C_{p}^{N D}$ and $C_{p}^{m}$ since it does not take into consideration the correlation between the two quality characteristics and therefore, it has the tendency of overestimate the capability of the process. On the other hand, Niverthi and Dey's has the tendency of underestimate the capability. Mingoti and Glória's resulted in values in between both and it was able to describe more properly the true capability of the processes considered. Also in all cases the values of the capability multivariate indexes are functions of the confidence level $(1-\alpha), 0<\alpha<1$. Depending of the choice of $\alpha$ the process might be considered capable or not. Therefore, the choice of $\alpha$ is very important to evaluate the process capability.

\subsection{Simulation}

A total of $k=100$ random samples of size $n=100$ were generated for each simulated process. Niverthi and Dey's multivariate PCI and Mingoti and Glória's index were calculated for each sample considering $\alpha=0.0027$. Table 3 presents the average and the standard deviation of the PCI's estimates for process 1. The estimated Niverthi and Dey's PCI resulted in coefficients that are very similar to their corresponding $C_{p}^{N D}$ theoretical values for each variable and with small standard deviations. The same occurred with Mingoti and Glória's index estimates which were similar to the theoretical $C_{p}^{m}$ values. The results for the multivariate Niverthi and Dey's $\hat{C}_{p k}^{N D}$ and Mingoti and Glória's $\hat{C}_{p k}^{m}$ will not be shown because they were very similar to those obtained for $\hat{C}_{p}^{N D}$ and $\hat{C}_{p}^{m}$ since the process 1 is centered in the specification mean vector. Table 4 presents the $\hat{C}_{p k}^{N D}$ and $\hat{C}_{p k}^{m}$ average values for the process 2 for $\alpha=0.0027$. The fit was also good as 
expected. The $\hat{C}_{p}^{m}$ resulted in smaller standard deviations in both cases. Therefore, this simulation study indicated that the estimators of the multivariate Niverthi and Dey's (2000) and Mingoti and Glória’s (2003) indexes described well the true theoretical values of the multivariate process capability corresponding to each methodology. Mingoti and Glória’s had better performance since it presented smaller mean error and standard deviation in both processes.

\section{CONFIDENCE INTERVALS FOR CAPABILITY INDEXES USING BOOTSTRAP METHODOLOGY}

The bootstrap methodology (EFRON; TIBSHIRANI,1993) can be used to generate confidence intervals for the true process capability indexes for each methodology. Given a sample of size $n$ of the process $m$ random samples with replacement are selected from this sample, called bootstrap samples. For each bootstrap sample the $C_{p}^{N D}, C_{p}^{m}, C_{p k}^{N D}$ and $C_{p k}^{m}$ are estimated and their sample distribution are obtained. A confidence interval for the true capability values can be obtained by using methods such as percentile, the accelerated bias-corrected, the bias-corrected percentile and t-bootstrap methods (GARTHWAITE et al.,1995). As an illustration we will return to the aircraft example presented in section 7. Considering the original sample of size $n=50$ presented in Niverthi and Dey's paper (2000) for those $p=4$ variables of the example, a total of $m=500$ bootstrap samples were selected with replacement. For each bootstrap sample the estimates $\hat{C}_{p}^{N D}$ and $\hat{C}_{p}^{m}$ calculated using $\alpha=0.0027$, were compared to the values of the vector $\hat{C}_{p}^{N D}$ given in (17) and to $C_{p}^{m}=0.783$ value given in (19). Table 5 shows the Mean Error $(M E)$ and the Squared Mean Error $(S M E)$ resulted from this comparison. For Niverthi and Dey the $M E$ and $S M E$ values are averages of the corresponding values calculated for each of the 4 variables. As we can see the errors were larger for Niverthi and Dey estimates than for Mingoti and Glória's index which had a very good fit and was practically unbiased. The 95\% confidence limits obtained for Niverthi and Dey's and Mingoti and Glória's PCI true process indexes are given in Table 6. If the univariate capability reference values were considered for a comparison we could conclude that the process is capable for the first, third and fourth variables but might be incapable for the second variable since the confidence interval includes values lower than 1 according to Niverthi and Dey's. The confidence interval obtained according to Mingoti and Glória’s capability index indicates that the process is incapable. Also, the confidence interval using Mingoti and Glória's index resulted in smaller range than Niverthi and Dey's.

Table 3: Descriptive statistics of the capability estimates - Process $1-\alpha=0.0027$.

\begin{tabular}{cccc}
\hline $\begin{array}{c}\text { Descriptive } \\
\text { statistics }\end{array}$ & ND- first variable & ND- second variable & MG \\
& $\hat{C}_{p}$ & $\hat{C}_{p}$ & $\hat{C}_{p}^{m}$ \\
Mean & 1.407 & 0.899 & 1.425 \\
Median & 1.415 & 0.897 & 1.423 \\
Standard deviation & 0.124 & 0.132 & 0.102 \\
Variation Coefficient & 0.088 & 0.147 & 0.072 \\
\hline
\end{tabular}

(*) True values of $C_{p}=\left[\begin{array}{ll}1.412 & 0.885\end{array}\right] ; C_{p}^{m}=1.429 ;$ ND:Niverthi \& Dey; MG:Mingoti \& Glória.

Table 4: Descriptive statistics of the capability estimates - Process $2-\alpha=0.0027$.

\begin{tabular}{cccc}
\hline $\begin{array}{c}\text { Descriptive } \\
\text { statistics }\end{array}$ & ND- first variable & ND-second variable & MG \\
Mean & $\hat{C}_{p k}$ & 0.439 & $\hat{C}_{p k}^{m}$ \\
Median & 1.635 & 0.7463 & 0.777 \\
Standard deviation & 1.626 & 0.101 & 0.004 \\
Variation Coefficient & 0.141 & 0.229 & 0.005 \\
\hline
\end{tabular}

(*) True values of: $C_{p k}=\left[\begin{array}{ll}1.609 & 0.431\end{array}\right] ; C_{p k}^{m}=0.783 ;$ ND:Niverthi \& Dey; MG:Mingoti \& Glória. 
In this section only the confidence intervals for the true Mingoti and Glória global capability index was presented which corresponds to the variable with lower capability. However, by using the bootstrap methodology it is possible to obtain confidence intervals for Mingoti and Glória’s true capability of each quality characteristic individually.

\section{FINAL REMARKS}

The examples presented in this paper show that the Mingoti and Glória's capability index $\left(C_{p}^{m}\right)$, which is a modification of Chen's capability coefficient (1994), is more precise than Niverthi and Dey's $\left(C_{p}^{N D}\right)$ and less biased. The $C_{p}^{m}$ produces a capability value for each variable and a global capability index differently than Niverthi and Dey which was originally proposed to give only a capability coefficient for each variable separatedly. In this paper we introduced the idea of measuring the global capability by taking Niverthi and Dey's vector minimum value. By using Hayter and Tsui (1994) methodology, the calculation of Mingoti and Glória’s capability index is more feasible for any number $p$ of variables. This is because the calculation will depend only of a simple simulation procedure used to obtain the constant $C_{R \alpha}$ related to the distribution of the maximum of the coordinates of a random vector with $p$-variate normal distribution. If the distribution is not multivariate normal the $C_{p}^{m}$ capability index still can be used since the constant $C_{R \alpha}$ can be obtained by a non-parametric procedure as suggested in Hayter and Tsui (1994) or by Kernel methodology (POLANSKY; BAKER, 2000; GLÓRIA, 2006). The same is not true for Niverthi and Dey's indexes. It is also important to point out that in the examples presented in this paper the value of the geometric mean was always higher than Mingoti and Glória's and Niverthi and Dey's overestimating the true process capability. On the other hand, Niverthi and Dey's penalizes the process more than Mingoti and Glória's indicating sometimes that the process is not capable when it really is. For the examples presented in this paper Mingoti and Glória's described more properly the true capability of the processes. Finally, the bootstrap methodology is an interesting alternative to produce confidence intervals for the true capability indexes of multivariate processes.

Table 5: Mean error and square mean error values - Niverthi \& Dey and Mingoti \&

Glória capability indexes estimates - Bootstrap example $(p=4)-\alpha=0.0027$.

\begin{tabular}{ccccc}
\hline Descriptive statistics & ME & SME & ME & SME \\
& Niverthi \& Dey & Niverthi \& Dey & Mingoti \& Glória & Mingoti \& Glória \\
\hline Mean & 0.1752 & 0.0971 & -0.0069 & 0.0032 \\
Standard deviation & 0.1135 & 0.0594 & 0.0563 & 0.0063 \\
\hline
\end{tabular}

Table 6: Bootstrap 95\% confidence intervals for the true capability indexes.

\begin{tabular}{cccccc}
\hline $\begin{array}{c}\text { Confidence } \\
\text { Interval }\end{array}$ & $\begin{array}{c}\text { ND } \\
\text { Variável 1 }\end{array}$ & $\begin{array}{c}\text { ND } \\
\text { Variável 2 }\end{array}$ & $\begin{array}{c}\text { ND } \\
\text { Variável 3 }\end{array}$ & $\begin{array}{c}\text { ND } \\
\text { Variável 4 }\end{array}$ & Mingoti and Glória \\
\hline Lower limit & 2.187 & 0.809 & 1.571 & 1.669 & 0.691 \\
Upper limit & 3.258 & 1.236 & 2.493 & 2.489 & 0.909 \\
Interval range & 1.071 & 0.427 & 0.922 & 0.820 & 0.218 \\
\hline
\end{tabular}

(*) ND: Niverthi \& Dey Capability index. 


\section{REFERENCES}

BERNARDO, J. M.; IRONY, T. X. A general multivariate bayesian process capability index. Statistician, v. 45, n. 3, p. 487-502, 1996.

CHEN, H. A multivariate process capability index over a rectangular solid tolerance zone, Statistica Sinica, v. 4, n. 2, p. 749-758, 1994.

CHENG, S. W.; SPIRING, F. A. Assessing process capability: a bayesian approach. IEE Transactions, 21, p. 97-98, 1989.

EFRON, B.; TIBSHIRANI, R. J. An Introduction to the Bootstrap. New York: Chapman and Hall, 1993.

FOSTER, E. J.; BARTON, R. R.; GAUTAM, N.; TRUSS, L. T.; TEW, J. D. The process-oriented multivariate capability index. International Journal of Production Research, v. 43, n.10, p. 2135-2148, 2005

GARTHWAITE, P. H.; JOLLIFFE, I. T.; JONES, B. Statistical inference. New York: Prentice Hall, 1995.

GLÓRIA, F. A. A. Uma avaliação do desempenho de núcleo-estimadores no controle de processos multivariados. Dissertação (Mestrado em Estatística). Universidade Federal de Minas Gerais, Departamento de Estatística. Belo Horizonte, 2006.

HAYTER, A. J.; TSUI, K-L. Identification and quantification in multivariate quality control problems. Journal of Quality Technology, v. 26, n. 3, p. 197-208, 1994.

JOHNSON, R. A.; WICHERN, D. W. Applied Multivariate Statistical Analysis. New Jersey: Prentice Hall, 2002.

KALGONDA, A. A.; KULKARNI, S. R. Multivariate quality control chart for autocorrelated pro- cesses. Journal of Applied Statistics, v. 31,n. 3, p. 317-327, 2004.

KOTZ, S.; JOHNSON, N. L. Process capability indexes-a review, 1992-2000. Journal of Quality Technology, v. 34, n. 1, p. 2-39, 2002.

MASON, R. L.; YOUNG, J. C. Multivariate Statistical Process Control with Industrial Applications. Pennsylvania: Siam and ASA, 2002.

MINGOTI, S. A.; GLÓRIA, F. A. A. Comparando os métodos paramétrico e não-paramétrico na determinação do valor crítico do teste estatístico de médias proposto por Hayter e Tsui. Produção, v. 15, n. 2, p. 251-262, 2005.

MINGOTI, S. A.; GLÓRIA, F. A. A. Uma proposta de modificação do índice de capacidade multivariado de Chen. In Anais do XXIII ENEGEP, Ouro Preto, Minas Gerais, 2003 (em cd-rom).

MONTGOMERY, D. C. Introduction to Statistical Quality Control. New York: John Wiley, 2001.

NIVERTHI, M.; DEY, D. K. Multivariate process capability: a bayesian perspective. Communications in Statistics-Simulation and Computation, 29, p. 667-687, 2000.

PEARN, W. L.; WANG, F. K.; YEN, C. H. Multivariate capability indices: distributional and inferential properties. Journal of Applied Statistics, v. 34, n. 8, p. 941-962, 2007.

PEARN, W. L.; WU, C. W. Production quality and yield assurance for processes with multiple independent characteristics. European Journal of Operational Research, v. 173, n. 2, p. 637-647, 2006

POLANSKY, A. M. A smooth nonparametric approach to multivariate process capability. Technometrics, v. 43, n. 2, p. 199-211, 2001.
POLANSKY, A. M.; BAKER, E. R. Multistage plug-in bandwidth selection for kernel distribution function estimates. Journal of Statistical Computation and Simulation, v. 65, n. 1, p. 63-80, 2000.

SHAHRIARI, H.; HUBELE, N. F.; LAWRENCE, F. P. A multivariate process capability vector In Proceedings of the 4th Industrial Engineering Research Conference, Institute of Industria Engineers, p. 305-309, 1995.

TAAN, W.; SUBBAIHA, P.; LIDDY, J. W. A note on multivariate capability indexes. Journal of Applied Statistics, v. 20, n. 3, p. 339-351, 1993.

TAAN, P. F.; BARNETR, N. S. Capability indexes for multivariate processes. Technical report. Division of computation Mathematics and Science. Victoria University. Melbouurne, Australia, 1998.

VEEVERS, A. Viability and capability indexes for multiresponse processes. Journal of Applied Statistics, v. 25, n. 4, p. 545-558, 1998.

WANG, C. H. Constructing multivariate process capability indices for short-run production. International Journal of Advanced Manufacturing Technology, 26, p. 1306-1311, 2005

WANG, F. K. Quality evaluation of a manufactured product with multiple characteristics. Quality and Reliability Engineering International, v. 22 , n. 2, p. 225-236, 2006

WANG, F. K.; HUBELE, N.F.; LAWRENCE, F. P.; MISKUKIN, J. O.; SHARIARI, H. Comparison of three multivariate process capability indexes. Journal of Quality Technology, v. 32, n. 3, p. 263-275, 2000

ZHANG, N. Z. Estimating process capability indexes for autocorrelated data. Journal of Applied Statistics, v. 25, n. 4, p. 559-574, 1998.

\section{ACKNOWLEDGMENT}

The authors are very grateful to the unknown referees for their helpful comments and suggestions

The authors were partially supported by the Brazilian Council for Scientific and Technological Development (CNPq). 
Mingoti, S. A.; Glória, F. A. A. Comparing Mingoti and Glória's and Niverthi and Dey's multivariate capability indexes. Produção, v. 18, n. 3 , p. $598-608,2008$

\section{ABOUT THE AUTHORS}

\section{Sueli Aparecida Mingoti}

Departamento de Estatística - ICEx.

Universidade Federal de Minas Gerais

End.: Av. Antonio Carlos, 6627 - Campus Pampulha - CEP 30161-970 - Belo Horizonte - Minas Gerais.

Tel.: (031) 3-4995948 - Fax: 3-499-5924

E-mail:sueliam@est.ufmg.br

\section{Fernando Augusto Alves Glória}

Nestle S/A

End.: Rua Henry Nestle, S/N - Vila Formosa - São José do Rio Pardo - SP - CEP 13720-000

E-mail: fernando.gloria@br.nestle.com
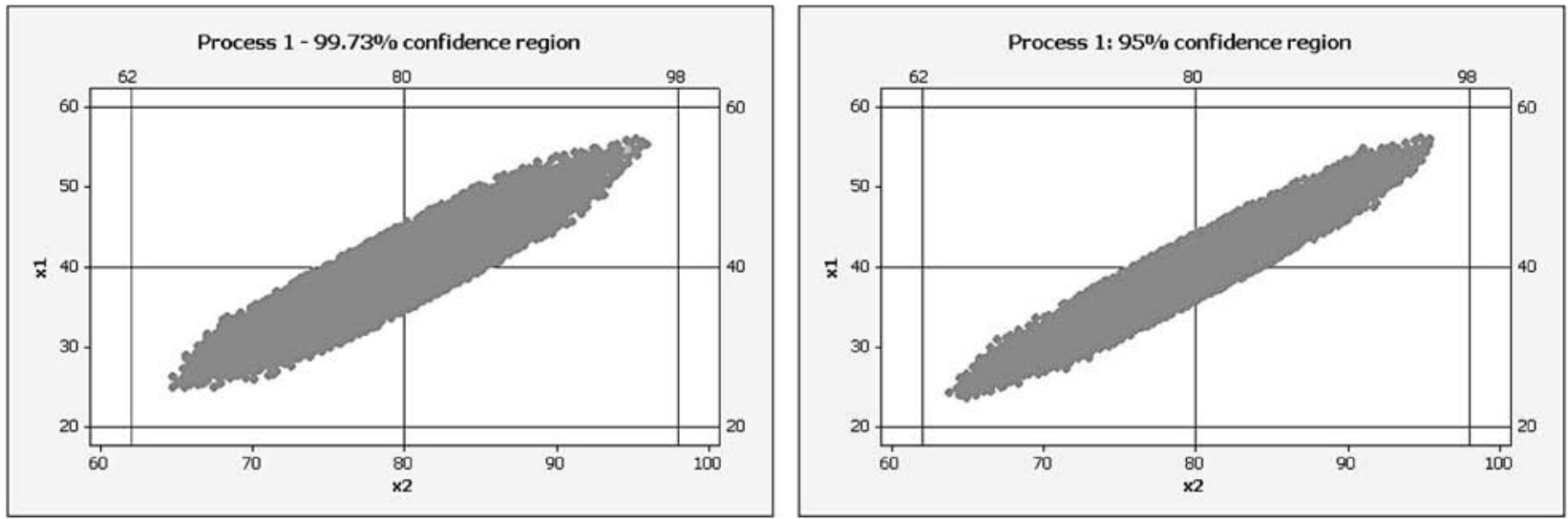

Figure 1: Confidence region and specification limits for process 1.
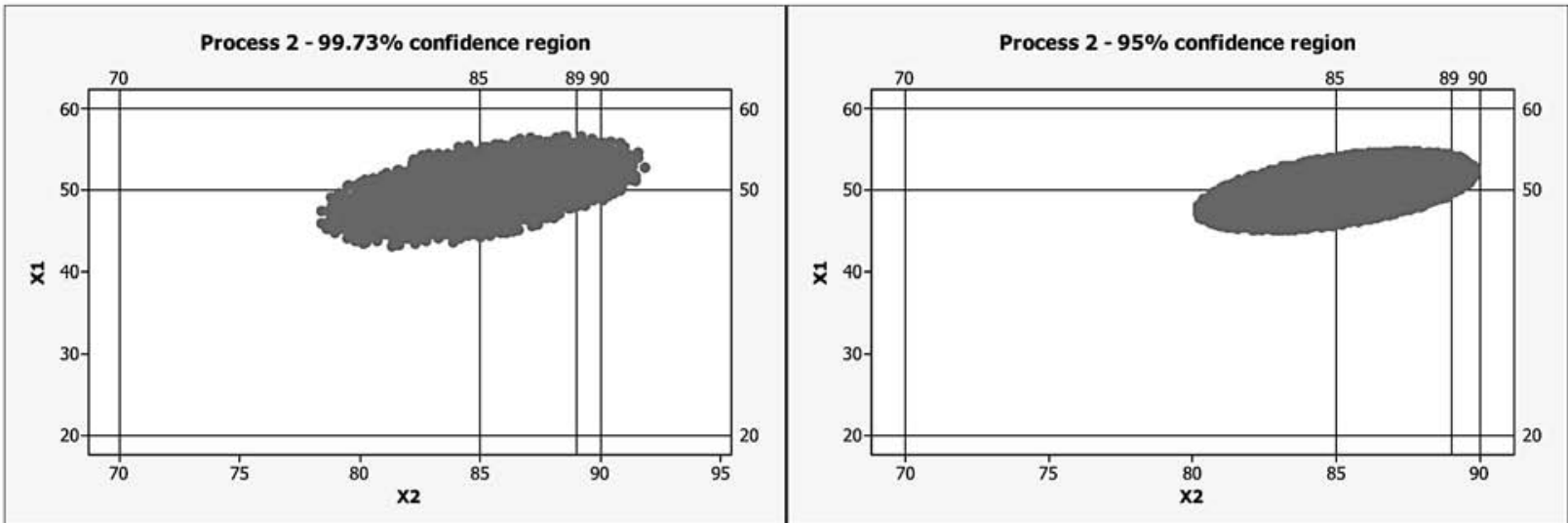

Figure 2: Confidence region and specification limits for process 2. 